\title{
Discovery of a single topological Dirac fermion in the strong inversion asymmetric compound $\mathrm{BiTeCl}$
}

\author{
Y. L. Chen ${ }^{1,2,3 \star}$, M. Kanou ${ }^{4}$, Z. K. Liu ${ }^{5}$, H. J. Zhang ${ }^{5}$, J. A. Sobota ${ }^{3,5}$, D. Leuenberger ${ }^{3,5}$, S. K. Mo ${ }^{6}$, \\ B. Zhou ${ }^{1,6}$, S-L. Yang ${ }^{3,5}$, P. S. Kirchmann ${ }^{3}$, D. H. Lu' , R. G. Moore ${ }^{3}$, Z. Hussain ${ }^{6}$, Z. X. Shen ${ }^{3,5}$, X. L. Qi ${ }^{5}$ \\ and T. Sasagawa ${ }^{4 \star}$
}

In the past few years, a new state of quantum matter known as the time-reversal-invariant topological insulator has been predicted theoretically and realized experimentally. All of the topological insulators discovered so far in experiment are inversion symmetric ${ }^{1-5}$-except for strained $\mathrm{HgTe}$, which has weak inversion asymmetry, a small bulk gap but no bulk charge polarization ${ }^{6}$. Strong inversion asymmetry in topological insulators would not only lead to many interesting phenomena, such as crystalline-surface-dependent topological electronic states, pyroelectricity and intrinsic topological p-n junctions, but would also serve as an ideal platform for the realization of topological magneto-electric effects ${ }^{7,8}$, which result from the modification of Maxwell equations in topological insulators. Here we report the discovery of a strong inversion asymmetric topological insulator phase in $\mathrm{BiTeCl}$ by angle-resolved photoemission spectroscopy, which reveals Dirac surface states and crystalline-surface-dependent electronic structures. Moreover, we observe a tenfold increase of the bulk energy gap in $\mathrm{BiTeCl}$ over the weak inversion asymmetric topological insulator $\mathrm{HgTe}$, making it a promising platform for topological phenomena and possible applications at high temperature.

Topological insulators represent a new state of quantum matter with a bulk energy gap and robust surface states formed by an odd number of Dirac fermions with helical spin texture. The gaplessness of the surface states is protected by the time-reversal symmetry. Depending on their crystal structure, topological insulators may or may not preserve inversion symmetry. Although the presence of inversion symmetry is helpful in identifying topological insulators owing to the existence of the parity criterion ${ }^{9}$, the search for inversion asymmetric topological insulators (IATIs) persists $^{8,10-13}$ as an effort to realize new topological phenomena in practical material systems.

IATIs have many unusual properties. For example, in an IATI, the top and bottom crystal surfaces are in-equivalent, resulting in different surface electronic structures. If the charge carriers of different surface states are opposite (Fig. 1a), a natural topological $\mathrm{p}-\mathrm{n}$ junction is formed (Fig. 1a), enabling chiral edge states in a magnetic field that can carry dissipationless transport ${ }^{14}$. Furthermore, when an IATI is driven to the topological phase transition towards a trivial insulator, Weyl semimetals can be realized at the phase transition with interesting topological properties ${ }^{15,16}$; and the broken inversion symmetry may also lead to topological superconductivity if an IATI is driven to a superconducting phase ${ }^{17}$. IATIs are also ideal candidates for the realization of the topological magneto-electric effects $\mathrm{s}^{8,10}$ (TMEs), which requires breaking both the inversion and timereversal symmetries. For a usual inversion symmetric topological insulator (ISTI), this demands opposite magnetization on opposite surfaces (Fig. 1b,d), posing a great challenge for the experimental realization. On the contrary, in an IATI, a uniform magnetic field (or ferromagnetic order) will be sufficient to induce the TME effects (Fig. 1c,e) as the inversion symmetry is naturally broken by the crystal structure.

So far, all topological insulators experimentally discovered preserve inversion symmetry-such as the $\mathrm{Bi}_{1-x} \mathrm{Sb}_{x}$ alloy ${ }^{1}, \mathrm{~V}_{2}-\mathrm{VI}_{3}$ family compounds $\left(\mathrm{Bi}_{2} \mathrm{Se}_{3}, \mathrm{Bi}_{2} \mathrm{Te}_{3}\right.$ and $\mathrm{Sb}_{2} \mathrm{Te}_{3} ;$ refs 2,3) and III-V-VI ${ }_{2}$ family compounds (for example, TlBiSe ${ }_{2}$; refs 4,5), except for strained $\mathrm{HgTe}$ (ref. 6), which is inversion asymmetric owing to its zinc blende structure. However, the inversion asymmetry in HgTe is weak in the sense that the crystal shows no net charge polarization (as we will discuss later). In addition, the small bulk bandgap $(\sim 20 \mathrm{meV})$ of $\mathrm{HgTe}$ induced by strain ${ }^{6}$ severely limits the temperature under which new topological phenomena can be observed.

Here, we studied two v-vi-vil compounds (BiTeCl and BiTeI) using angle-resolved photoemission spectroscopy (ARPES) to investigate their electronic structures. In both materials, we found that the electronic structures from opposite crystal surfaces (with halogen or chalcogen termination, respectively) are significantly different as a result of the strong inversion asymmetry. In fact, the difference is so pronounced that the charge carriers on the two surfaces become opposite types, leading to strong bulk charge polarization. Surprisingly, for $\mathrm{BiTeCl}$, we observed a single surface Dirac cone inside the bulk energy gap, making it the first strong inversion asymmetric polar (and also pyroelectric) topological insulator candidate. Furthermore, the bulk energy gap observed in $\mathrm{BiTeCl}$ was $\sim 220 \mathrm{meV}$, showing a tenfold increase over that for strained $\mathrm{HgTe}$ (refs 6,18; $\sim 20 \mathrm{meV}$ ). For BiTeI, we observed no topological surface states; however, in addition to the Rashba splitting in its bulk conduction band (BCB) recently reported ${ }^{19,20}$, we found that the bulk valence band (BVB) also has Rashba

\footnotetext{
${ }^{1}$ Clarendon Laboratory, Department of Physics, University of Oxford, Parks Road, Oxford OX1 3PU, UK, ${ }^{2}$ Diamond Light Source, Didcot OX11 ODE, UK, ${ }^{3}$ Stanford Institute for Materials and Energy Sciences, SLAC National Accelerator Laboratory, 2575 Sand Hill Road, Menlo Park, California 94025, USA, ${ }^{4}$ Materials and Structures Laboratory, Tokyo Institute of Technology, Kanagawa 226-8503, Japan, ${ }^{5}$ Geballe Laboratory for Advanced Materials,

Departments of Physics and Applied Physics, Stanford University, Stanford, California 94305, USA, ${ }^{6}$ Advanced Light Source, Lawrence Berkeley National Laboratory, Berkeley, California 94720, USA, 7 Stanford Synchrotron Radiation Lightsource, 2575 Sand Hill Road, California 94025, USA.

*e-mail:Yulin.Chen@physics.ox.ac.uk; sasagawa@msl.titech.ac.jp
} 
a
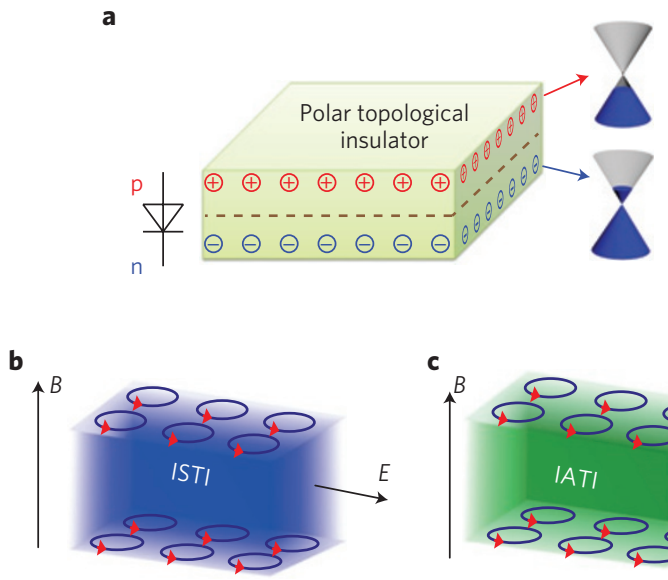

d

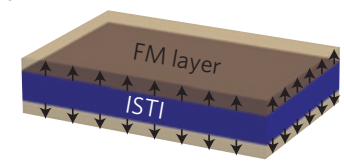

c

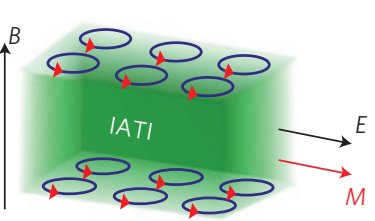

e

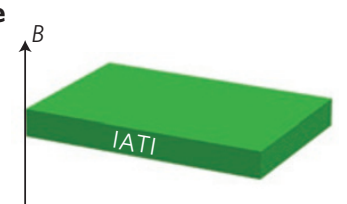

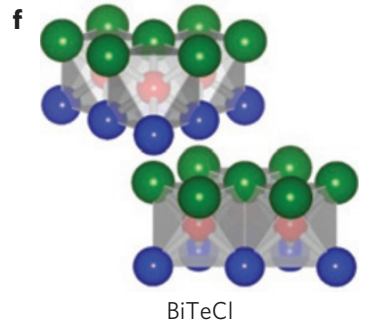

h

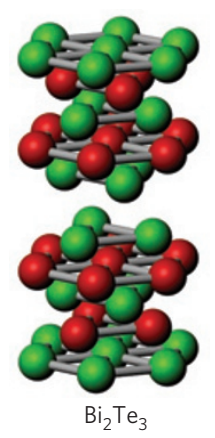

g

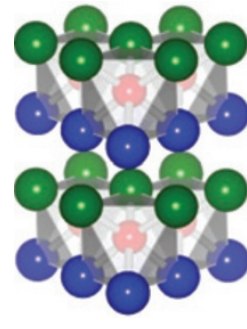

BiTel

i

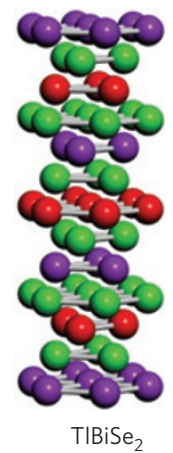

Figure 1 | Introduction and applications of IATIs. a, Illustration of a topological p-n junction formed by top and bottom surfaces of a polar topological insulator. The different $E_{\mathrm{F}}$ positions of the top and bottom Dirac cones are shown in the inset. b,c, Under a uniform magnetic field, an electric field $E$ does not induce a magnetic moment in an ISTI (b), whereas it does introduce a magnetic moment $M$ in an IATI (c). FM, ferromagnetic. d,e, Experimental set-up for realizing the TME effect in an ISTI (d) and an IATI (e). f,g, Crystal structures of inversion asymmetric compounds BiTeCl (f) and BiTel (g); the two

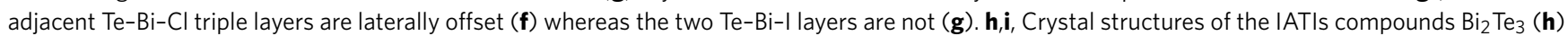
and $\mathrm{TIBiSe}_{2}(\mathbf{i})$.

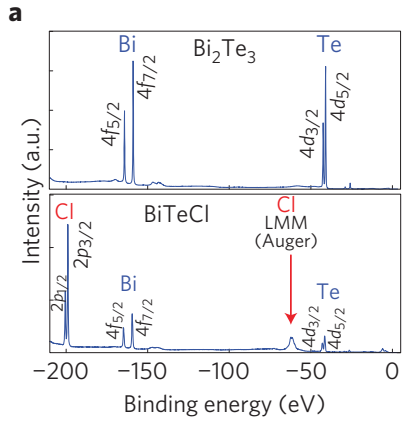

b

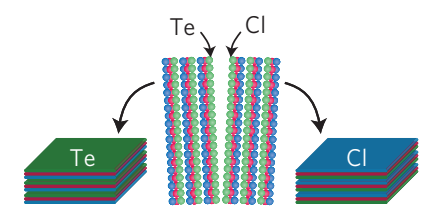

c

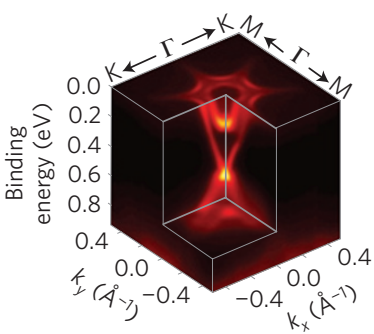

d

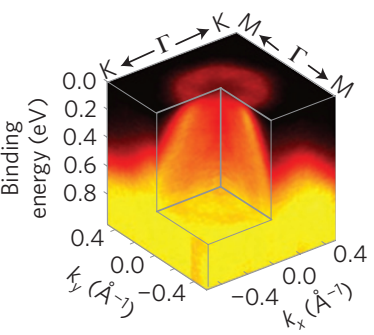

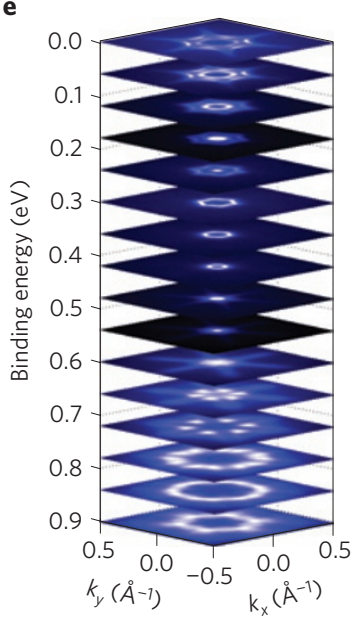

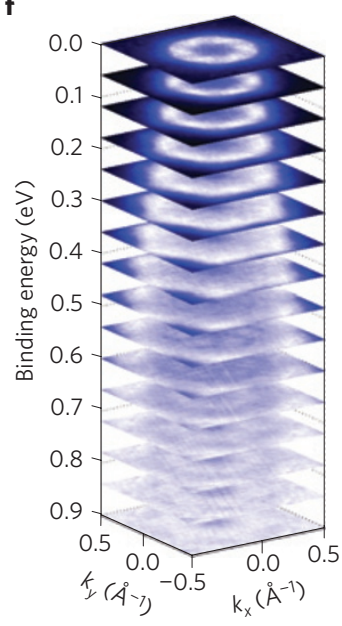

Figure 2 | Asymmetric band structures from opposite crystal surfaces of $\mathrm{BiTeCl} . \mathbf{a}_{1}$ Comparison between the core-level photoemission spectra of $\mathrm{Bi}_{2} \mathrm{Te}_{3}$ (upper panel) and $\mathrm{BiTeCl}$ (lower panel), which clearly show the $4 d / 4 f$ level peaks of $\mathrm{Te} / \mathrm{Bi}$ and $2 p$ level peaks of $\mathrm{Cl}$, as well as the $\mathrm{LMM} \mathrm{Auger} \mathrm{peak} \mathrm{from} \mathrm{Cl}$. b, Illustration of the method used in experiments to obtain a pair of surfaces with different terminations from the same crystal. c,d, $3 D$ intensity plot of the photoemission spectra measured from a pair of surfaces obtained in $\mathbf{b}$, showing an $\mathrm{n}$ - (top panel) and a p-type (bottom panel) band structure, respectively. $\mathbf{e}, \mathbf{f}$, Plots of constant energy contours of the two types of band structure in $\mathbf{c}, \mathbf{d}$, respectively. Separation between the consecutive contours is $0.06 \mathrm{eV}$. The size and the shape of the Fermi surface in $\mathbf{f}$ is similar to the contours between 0.8 and $0.9 \mathrm{eV}$ binding energy in $\mathbf{e}$.

splitting, suggesting that both $\mathrm{n}$ - and p-type BiTeI may be good candidates for spintronic applications.

The crystal structures of BiTeCl and BiTeI are shown in Fig. 1f,g. Despite the subtle difference in crystal symmetry ( $\mathrm{BiTeCl}$ being $P 63 m c$ and BiTeI being $P 3 m 1$ ), both compounds are inversion asymmetric, distinct from the $\mathrm{v}_{2}-\mathrm{VI}_{3}$ (for example, $\mathrm{Bi}_{2} \mathrm{Te}_{3}$, Fig. 1h) and $\mathrm{II}-\mathrm{V}-\mathrm{VI}_{2}$ (for example, $\mathrm{TlBiSe}_{2}$, Fig. 1i) topological insulator compounds ${ }^{2-5,21,22}$.
When comparing with $\mathrm{Bi}_{2} \mathrm{Te}_{3}$, the presence of the $\mathrm{Cl}$ element in $\mathrm{BiTeCl}$ is obvious in the core-level photoemission spectra (Fig. 2a). Owing to the broken inversion symmetry and the markedly different electron affinity between Te and $\mathrm{Cl}\left(190\right.$ and $349 \mathrm{~kJ} \mathrm{~mol}^{-1}$, respectively ${ }^{23}$ ), the electric charge in the crystal is expected to distribute unevenly along the $\mathrm{Te}-\mathrm{Bi}-\mathrm{Cl}$ layers. To examine this difference, for each single crystal, we conducted ARPES measurements on a pair of opposite crystal surfaces (Fig. 2b) for comparison. 


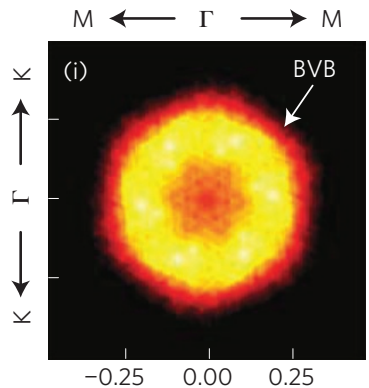

b
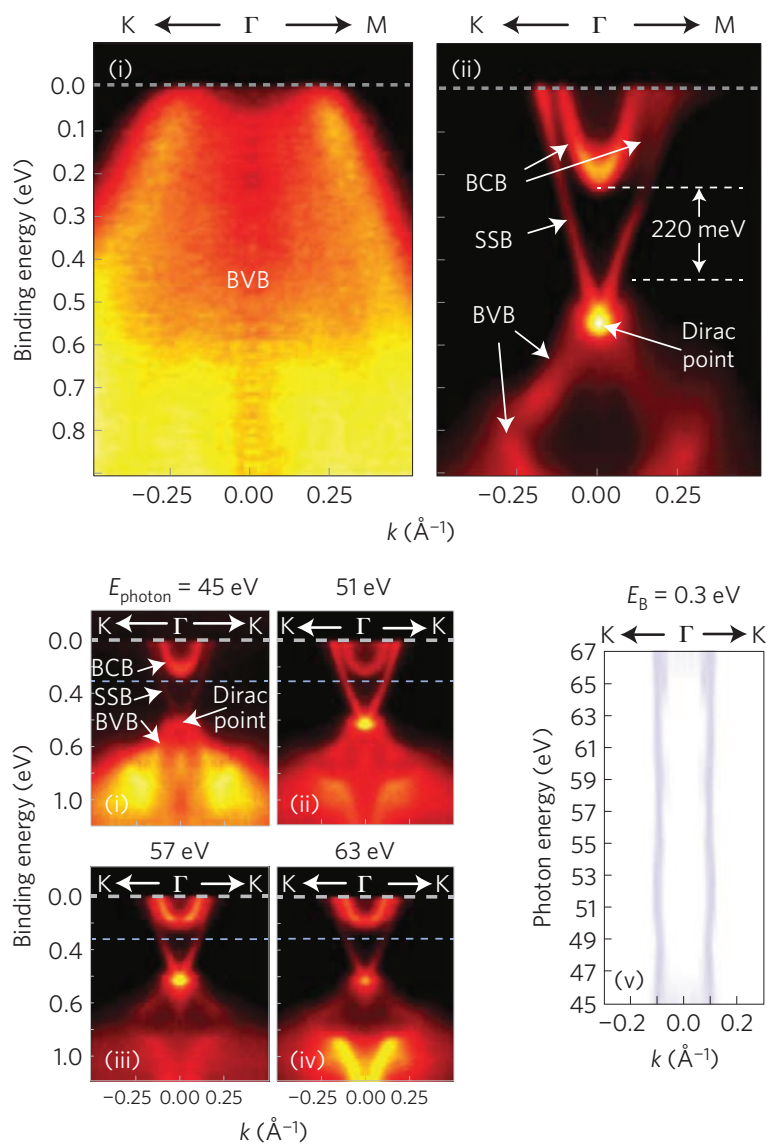

$\mathrm{M} \longleftarrow \Gamma \longrightarrow M$

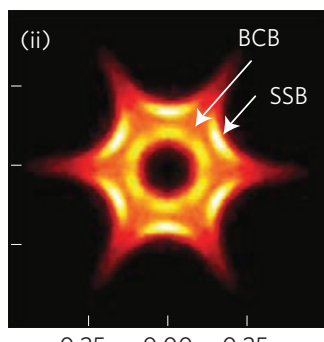

$-0.25 \quad 0.00 \quad 0.25$

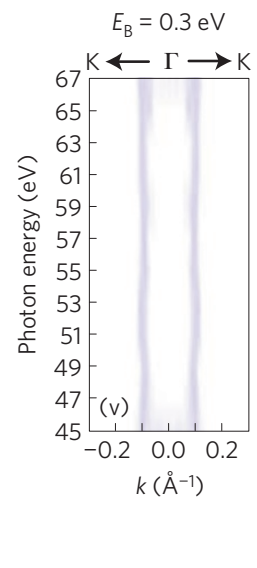

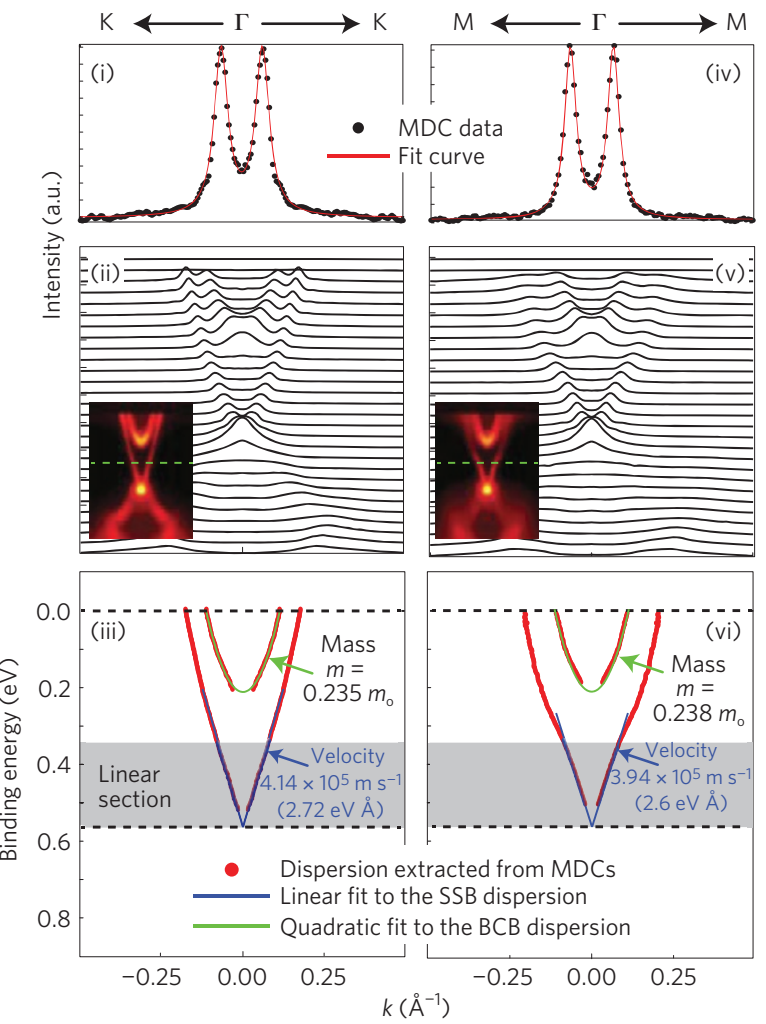

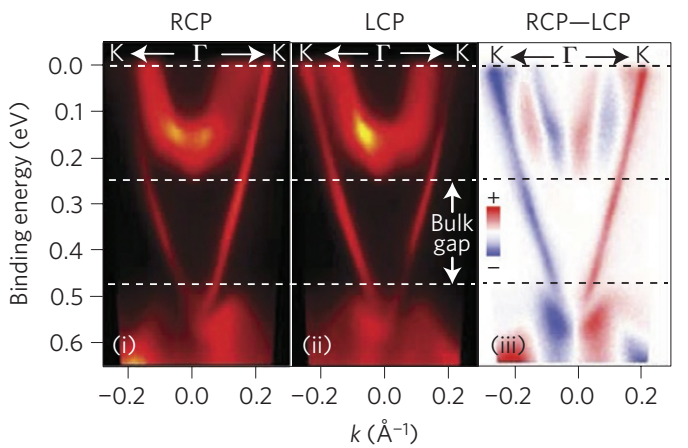

Figure $\mathbf{3}$ | Detailed band dispersions of BiTeCl. a,b, Fermi-surface plots (a) and the band dispersions (b) along the $\mathrm{K}-\Gamma-\mathrm{M}$ direction of the band structures measured on different termination surfaces of $\mathrm{BiTeCl}$. In both plots, the left panels (i) are from the p-type surface and the right panels (ii) are from the n-type surface. $\mathbf{c}$, Band dispersions extracted from the momentum distribution curve (MDC) line-shape analysis. $\mathbf{c}(\mathrm{i})$, An example shows the two Lorentzian-peak fit of an MDC at $E_{b}=0.4 \mathrm{eV}$, indicated by a green dashed line in $\mathbf{c}(\mathrm{ii})$. $\mathbf{c}(\mathrm{ii})$, Photoemission intensity plot (inset) and the MDC stack plots of the band dispersion along $\mathrm{K}-\Gamma-\mathrm{K}$ (left). c(iii), Bulk and surface dispersions extracted from MDC line shape analysis (red dots). Linear fits (blue line) are used to determine the Dirac fermion velocity $\left(4.14 \times 10^{5} \mathrm{~m} \mathrm{~s}^{-1}\right)$ and Dirac point energy position $(0.56 \mathrm{eV}$ ); and a quadratic fit on the bulk band (green line) indicate the bulk electron pocket effective mass as $0.2 m_{\mathrm{e}} \mathbf{c}$ (iv-vi): similar results as in $\mathbf{a}$ (i-iii) for the dispersion along the $\mathrm{M}-\Gamma-\mathrm{M}$ direction. d, Photon-energy-dependent ARPES measurement. d(i-iv), Example dispersions along the $\mathrm{K}-\Gamma-\mathrm{K}$ direction at four photon energies (45, 51,57 and $63 \mathrm{eV}$ ). $\mathbf{d}(\mathrm{V})$, Intensity plot of MDCs (at binding energy $E_{\mathrm{b}}=0.3 \mathrm{eV}$, for example indicated as blue dashed lines in panels i-iv) from the photon energy range 45-67 eV. e, Circular dichroism of ARPES spectra. e(i,ii), K- $\Gamma-\mathrm{K}$ dispersion for the right and left circularly polarized (RCP and LCP) photons, showing clear right-left asymmetry. $\mathbf{e}$ (iii), The dichroic spectra (RCP-LCP) clearly show the opposite spin polarization of the in-gap topological surface state for the positive and negative momenta. SSB, surface state band.

The measurement results from such a pair of surfaces are illustrated in Fig. 2c,d (measurements from more samples can be found in Supplementary Information, Part A). Evidently, the band structures from the two surfaces are markedly different, with Fig. 2c,d indicating n- and p-type carriers, respectively. These opposite carrier types from opposite crystal surfaces demonstrate a bulk charge polarization in $\mathrm{BiTeCl}$, making it a polar (and pyroelectric) material. By comparing the constant energy contours and the size of the Fermi momentum $\left(k_{\mathrm{F}}\right)$, we estimate that the difference in the Fermi energy $\left(E_{\mathrm{F}}\right)$ between the two crystal surfaces is approximately $0.8-0.9 \mathrm{eV}$ (Fig. $2 \mathrm{e}$,f: the Fermi surface and $k_{\mathrm{F}}$ in Fig. $2 \mathrm{f}$ resemble those at binding energy $0.8-0.9 \mathrm{eV}$ in Fig. 2e). This difference can also be seen in the detailed comparison between the Fermi surfaces and the band dispersions in Fig. 3a,b.

In addition to the difference in the carrier type between opposite crystal surfaces, there is a characteristic V-shaped linear dispersion in the n-type surface (Figs 2 and 3), forming a clear Dirac cone within the bulk energy gap ( $220 \mathrm{meV}$, see Fig. $3 \mathrm{~b}$ (ii). 


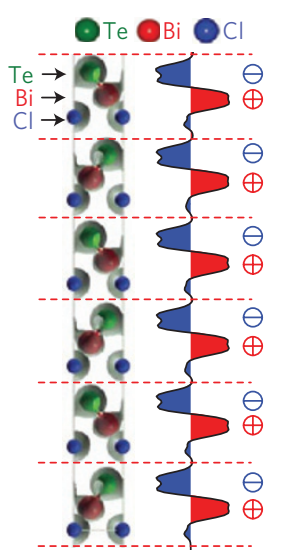

b

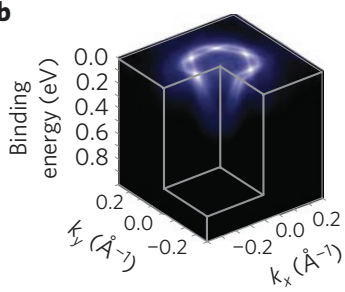

d

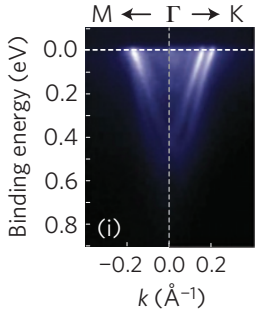

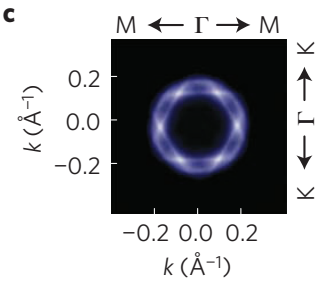

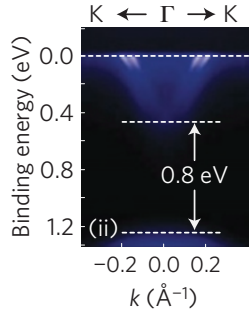

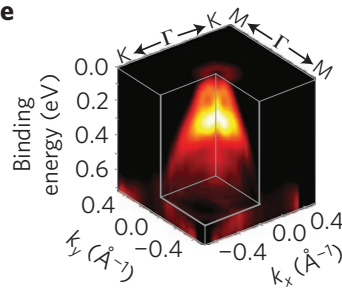

g
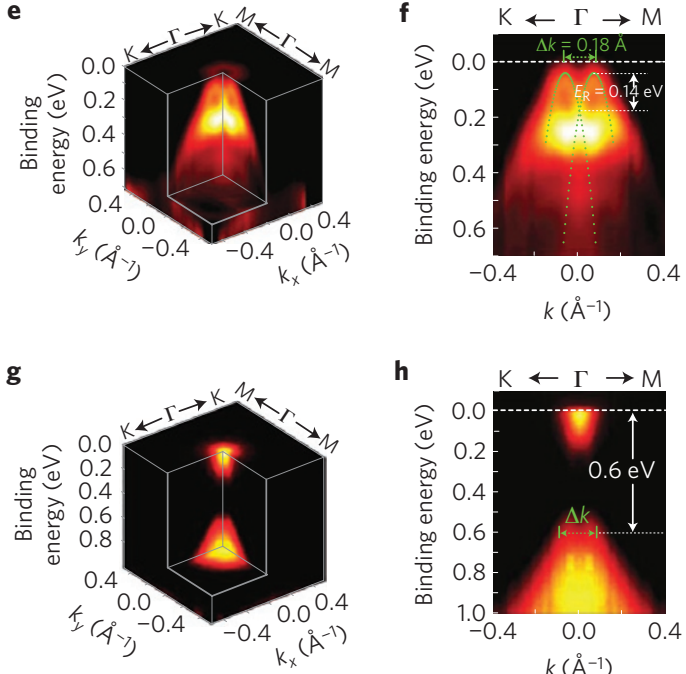

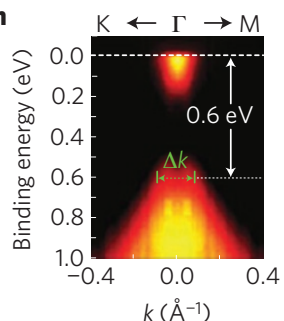

Figure 4 | Alternative band dispersions of $\mathrm{BiTeCl}$ and asymmetric band structures from opposite crystal surfaces of $\mathrm{BiTel}$. a, $\mathrm{Ab}$ initio calculation confirms the bulk band structure of $\mathrm{BiTeCl}$ that has clear bulk charge polarization. The left panel shows the charge distribution overlaid on the crystal structure; and the right panel is the integrated charge distribution as a function of c-axis location of BiTeCl. Red and blue filling show the positive and negative charge accumulation. b-d, Band structures occasionally observed on BiTeCl, showing the 3D ARPES spectra intensity plot in b, Fermi-surface plot in c and dispersions along high-symmetry directions in $\mathbf{d}$. e-h, Electronic structure of BiTel. e,f, 3D ARPES spectra intensity plot (e) and band dispersion along the $\mathrm{K}-\Gamma-\mathrm{M}$ high-symmetry direction (f) for the $\mathrm{p}$-type cleaved surface (I-termination, see text). By fitting the dispersion in $\mathbf{f}$, we get the Fermi momentum $k_{\mathrm{F}} \approx 0.09 \AA^{-1}$, the Rashba energy $E_{\mathrm{R}} \approx 0.14 \mathrm{eV}$ and the Rashba parameter $a_{\mathrm{R}} \approx 3.43$ eV $\AA$ (see Supplementary Information Part $\mathrm{H}$ for fitting details). $\mathbf{g}, \mathbf{h}, 3 D$ ARPES spectra intensity plot ( $\mathbf{g}$ ) and band dispersion along the $\mathrm{K}-\Gamma$-M high-symmetry direction (h) for the $n$-type cleaved surface (Te-termination, see text). Note that we observe no surface Dirac fermions in the bulk gap. In $\mathbf{h}$, the momentum position $k=0.09 \AA^{-1}$ (the $k_{\mathrm{F}}$ in $\mathbf{f}$ ) in the $B V B$ is located at the binding energy $E_{b}=0.6 \mathrm{eV}$.

More dispersions along all $k$-space directions can be found in Supplementary Movies S1 and S2), similar to that in other threedimensional (3D) topological insulators ${ }^{2-5,24}$. By fitting the linear dispersions (Fig. 3c), we obtained the velocity of the surface Dirac fermion as $4.14 \times 10^{5} \mathrm{~m} \mathrm{~s}^{-1}(2.73 \mathrm{eV} \AA)$ and $3.94 \times 10^{5} \mathrm{~m} \mathrm{~s}^{-1}$ $(2.6 \mathrm{eV} \AA$ ) along the $\Gamma-\mathrm{K}$ and $\Gamma-\mathrm{M}$ directions, respectively.

To prove the surface nature of the $\mathrm{V}$-shaped band, we performed photon-energy-dependent ARPES measurements ${ }^{25}$. In Fig. 3d, four dispersions (panels i-iv) are shown to illustrate the energy independence of the $\mathrm{V}$-shaped dispersion (results from more photon energies are available in Supplementary Information, Part B); and ARPES spectra over a broad photon energy range (45-67 eV, panel v) clearly show that the $\mathrm{V}$-shaped band has no $k_{z}$-dispersion, confirming its surface nature. Moreover, our ARPES circular dichroism measurements ${ }^{26}$ (Fig. $3 \mathrm{e}$ (iii), more details can be found in Supplementary Information, Part D) further illustrate that the in-gap V-shaped surface band is singly spin-degenerate; thus, its topological nature is fully established.

To associate the different charge carrier types to different crystal surfaces, we performed $a b$ initio calculations. The results in Fig. 4a clearly indicate the charge polarization within each $\mathrm{Te}-\mathrm{Bi}-\mathrm{Cl}$ triple layer unit, suggesting that the $n$ and $p$ types of surfaces originate from the Te and $\mathrm{Cl}$ terminations, respectively. This bulk polarization of $\mathrm{BiTeCl}$ is distinct from all previously discovered ISTIs (for example, $\mathrm{Bi}_{2} \mathrm{Te}_{3}, \mathrm{Bi}_{2} \mathrm{Se}_{3}$ or $\mathrm{TlBiTe}_{2}$ ) and weak IATI HgTe (see Supplementary Fig. S4). Interestingly, in addition to the band structures in Figs 2 and 3, we occasionally observed (once in several cleaves) very different band structures from BiTeCl (Fig. 4b-d) although the observed Rashba-split conduction bands resemble a recent calculation ${ }^{27}$, the experimental bandgap $(\sim 0.8 \mathrm{eV})$ is four times larger than the calculated value ${ }^{27}(\sim 0.2 \mathrm{eV})$. On the other hand, the results in Fig. 4b-d quantitatively agree with our ab initio calculations of a single-layer $\mathrm{BiTeCl}$ free-standing film (which may be produced during the cleaving process)—including the Fermi-surface, the band dispersion shape and the size of the energy gap magnitude (details of the cleaving process and the calculation results can be found in Supplementary Information, Part F).

Interestingly, the results for $\mathrm{BiTeCl}$ (Figs 2 and 3) show different electronic structures from the recent theoretical work ${ }^{27}$ that suggested it as a normal insulator. This difference may result from the fact that the calculation ${ }^{27}$ assumed that the crystal surfaces were passivated, whereas in the ARPES experiments, the samples were cleaved and measured in the ultrahigh vacuum environment that kept the surface clean during the measurements (see Supplementary Information, Part G). An estimation (Supplementary Information, Part G) shows that these surface charges may generate a large effective pressure $\left(\sim 10^{9} \mathrm{~Pa}\right)$ along the $c$ direction and thus drive the crystal (possibly the several top layers) into the topological insulator phase as suggested by a recent work $^{13}$. On the other hand, our measurements on BiTeI (Fig. $4 \mathrm{e}-\mathrm{h}$ ) of the same family generally agree with previous calculations $s^{13,27}$. The contrasting results from $\mathrm{BiTeCl}$ and $\mathrm{BiTeI}$ may motivate future theoretical studies and provide guidance for a refined theory that can explain their difference (for example, by considering the different crystal symmetry between $\mathrm{BiTeCl}$ and $\mathrm{BiTeI}$, the smaller $\mathrm{Cl}$ anion size and the larger electron affinity of $\mathrm{Cl}$ atoms).

Despite the difference above, the crystalline-surface-dependent electronic structure is also observed in BiTeI (Fig. $4 \mathrm{e}-\mathrm{h}$ ), with the $E_{\mathrm{F}}$ offset between different surfaces slightly less than that in $\mathrm{BiTeCl}$ ( $\sim 0.6 \mathrm{eV}$, Fig. $4 \mathrm{f}, \mathrm{h})$. This difference may result from the smaller electron affinity difference between Te and I (electron affinity of I and $\mathrm{Cl}$ is $\sim 55 \%$ and $\sim 84 \%$ stronger than Te, respectively ${ }^{23}$ ). In contrast to $\mathrm{BiTeCl}$, we did not observe in-gap topological surface states in BiTeI. Finally, besides the giant Rashba splitting recently discovered in the BCB (refs 19,20), we observed a giant Rashba splitting in the BVB (Fig. 4e,f) with a momentum offset $k_{0} \approx 0.09 \AA^{-1}$, Rashba energy $E_{\mathrm{R}} \approx 0.14 \mathrm{eV}$ and Rashba parameter $\alpha_{\mathrm{R}} \approx 3.4 \mathrm{eV} \AA$ (see Supplementary Information, Part H), making BiTeI a promising material for spintronic applications with both $\mathrm{p}$ - and n-type doping. 


\section{Methods}

Sample synthesis. Single crystals of $\mathrm{BiTeCl}$ were obtained by a self-flux growth method in an evacuated quartz tube. The starting material was a mixture of high-purity $(5 \mathrm{~N}) \mathrm{Bi}$, Te and $\mathrm{BiCl}_{3}$ at a molar ratio of 2:3:4, in which the excess $\mathrm{BiCl}_{3}$ serves as the self-flux. Owing to the deliquescence of $\mathrm{BiCl}_{3}$, weighing was done in a nitrogen-filled glovebox. The temperature profile of the crystal growth was optimized by the real-time observation of the sample space by using a home-made transparent two-zone tube furnace previously reported ${ }^{28}$. BiTeI crystals were grown from a stoichiometric mixture of $\mathrm{Bi}, \mathrm{Te}$ and $\mathrm{I}$ in an evacuated quartz tube by a modified Bridgman technique, similar to $\mathrm{Bi}_{2} \mathrm{Se}_{3}$ (ref. 24).

ARPES. ARPES measurements were performed at beam line 10.0.1 of the Advanced Light Source (ALS) at Lawrence Berkeley National Laboratory and beam line 5-4 of the Stanford Synchrotron Radiation Lightsource (SSRL). At both facilities, the measurement pressure was kept at $<2 \times 10^{-11}$ torr, and data were recorded by Scienta R4000 analysers at a $10 \mathrm{~K}$ sample temperature. The total convolved energy and angle resolutions were $10 \mathrm{meV} / 16 \mathrm{meV}$ and $0.2^{\circ} / 0.2^{\circ}$ (that is, $<0.007\left(\AA^{-1}\right)$ or $<0.012\left(\AA^{-1}\right)$ for photoelectrons generated by 20 and $48 \mathrm{eV}$ photons) at SSRL/ALS respectively. The fresh surface for ARPES measurement was obtained by cleaving the sample in situ along its natural cleavage plane.

Local-density approximation calculations. The $a b$ initio calculations were performed by the Vienna Ab initio Simulation Package ${ }^{29}$ within the framework of the Perdew-Burke-Ernzerhof-type generalized gradient approximation ${ }^{30}$ of the density functional theory. Spin-orbit coupling was included with the second-variational treatment with scalar-relativistic eigenfunctions as a basis. The energy cutoff was set to $350 \mathrm{eV}$. All of the calculations of charge distribution were carried out on bulk structures with experimental lattice parameters.

Received 24 February 2013; accepted 15 August 2013; published online 6 October 2013

\section{References}

1. Hasan, M. Z. \& Kane, C. L. Colloquium: Topological insulators. Rev. Mod. Phys. 82, 3045-3067 (2010).

2. Qi, X-L. \& Zhang, S-C. Topological insulators and superconductors. Rev. Mod. Phys. 83, 1057-1110 (2011).

3. Hsieh, D. et al. A topological Dirac insulator in a quantum spin Hall phase. Nature 452, 970-974 (2008).

4. Chen, Y. L. et al. Experimental realization of a three-dimensional topological insulator, $\mathrm{Bi}_{2} \mathrm{Te}_{3}$. Science 325, 178-181 (2009).

5. Xia, Y. et al. Observation of a large-gap topological-insulator class with a single Dirac cone on the surface. Nature Phys. 5, 398-402 (2009).

6. Chen, Y. L. et al. Single Dirac cone topological surface state and unusual thermoelectric property of compounds from a new topological insulator family. Phys. Rev. Lett. 105, 266401 (2010).

7. Kuroda, K. et al. Experimental realization of a three-dimensional topological insulator phase in ternary chalcogenide $\mathrm{TlBiSe}_{2}$. Phys. Rev. Lett. 105, 146801 (2010).

8. Brune, C. et al. Quantum Hall effect from the topological surface states of strained bulk HgTe. Phys. Rev. Lett. 106, 126803 (2011).

9. Qi, X-L., Hughes, T. L. \& Zhang, S-C. Topological field theory of time-reversal invariant insulators. Phys. Rev. B 78, 195424 (2008).

10. Fu, L. \& Kane, C. L. Topological insulators with inversion symmetry. Phys. Rev. B 76, 045302 (2007).

11. Chadov, S. et al. Tunable multifunctional topological insulators in ternary Heusler compounds. Nature Mater. 9, 541-545 (2010).

12. Yan, B. et al. Theoretical prediction of topological insulator in ternary rare earth chalcogenides. Phys. Rev. B 82, 161108 (2010).

13. Bahramy, M. S., Yang, B. J., Arita, R. \& Nagaosa, N. Emergence of non-centrosymmetric topological insulating phase in BiTeI under pressure. Nature Commun. 3, 679 (2012).

14. Wang, J., Chen, X., Zhu, B-F. \& Zhang, S-C. Topological p-n junction. Phys. Rev. B 85, 235131 (2012).
15. Xu, G., Weng, H., Wang, Z., Dai, X. \& Fang, Z. Chern semimetal and the quantized anomalous Hall effect in $\mathrm{HgCr}_{2} \mathrm{Se}_{4}$. Phys. Rev. Lett. 107, 186806 (2011).

16. Wan, X., Turner, A. M., Vishwanath, A. \& Savrasov, S. Y. Topological semimetal and Fermi-arc surface states in the electronic structure of pyrochlore iridates. Phys. Rev. B 83, 205101 (2011).

17. Qi, X-L., Hughes, T. L. \& Zhang, S-C. Topological invariants for the Fermi surface of a time-reversal-invariant superconductor. Phys. Rev. B 81, 134508 (2010).

18. Dai, X., Hughes, T. L., Qi, X-L., Fang, Z. \& Zhang, S-C. Helical edge and surface states in HgTe quantum wells and bulk insulators. Phys. Rev. B 77, 125319 (2008).

19. Ishizaka, K. et al. Giant Rashba-type spin splitting in bulk BiTeI. Nature Mater. 10, 521-526 (2011)

20. Landolt, G. et al. Disentanglement of surface and bulk Rashba spin splittings in noncentrosymmetric BiTeI. Phys. Rev. Lett. 109, 116403 (2012).

21. Zhang, $\mathrm{H}$. et al. Topological insulators in $\mathrm{Bi}_{2} \mathrm{Se}_{3}, \mathrm{Bi}_{2} \mathrm{Te}_{3}$ and $\mathrm{Sb}_{2} \mathrm{Te}_{3}$ with a single Dirac cone on the surface. Nature Phys. 5, 438-442 (2009).

22. Yan, B. et al. Theoretical prediction of topological insulators in thallium-based III-V-VI 2 ternary chalcogenides. Eur. Phys. Lett. 90, 37002 (2010).

23. Haynes, W. M. CRC Handbook of Chemistry and Physics 93rd edn (CRC Press, 2012).

24. Chen, Y. L. et al. Massive Dirac fermion on the surface of a magnetically doped topological insulator. Science 329, 659-662 (2010).

25. Chen, Y. L. Studies on the electronic structures of three-dimensional topological insulators by angle resolved photoemission spectroscopy. Front. Phys. 7, 175-192 (2012).

26. Wang, Y. \& Gedik, N. Circular dichroism in angle-resolved photoemission spectroscopy of topological insulators. Physica Status Solidi 7, 64-71 (2013).

27. Eremeev, S. V., Nechaev, I. A., Koroteev, Y. M., Echenique, P. M. \& Chulkov, E. V. Ideal two-dimensional electron systems with a giant Rashba-type spin splitting in real materials: Surfaces of bismuth tellurohalides. Phys. Rev. Lett. 108, 246802 (2012).

28. Iwasa, A. et al. Environmentally friendly refining of diamond-molecules via the growth of large single crystals. Cryst. Growth Des. 10, 870-873 (2009).

29. Kresse, G. \& Joubert, D. From ultrasoft pseudopotentials to the projector augmented-wave method. Phys. Rev. B 59, 1758-1775 (1999).

30. Perdew, J. P., Burke, K. \& Ernzerhof, M. Generalized gradient approximation made simple. Phys. Rev. Lett. 77, 3865-3868 (1996).

\section{Acknowledgements}

We thank Z. Wang and C. X. Liu for the helpful discussion. Y.L.C. acknowledges support from a DARPA MESO project (No. N66001-11-1-4105) and the EPSRC First Grant (EP/K04074X/1). B.Z., Z.K.L., Z.X.S. and X.L.Q. acknowledge support from Department of Energy, Office of Basic Energy Science (contract DE-AC02-76SF00515). T.S. acknowledges support from MEXT, Japan (Grant-in-Aid for Scientific Research (B), No. 24340078). H.J.Z. acknowledges support from the Army Research Office (No. W911NF-09-1-0508). J.A.S. acknowledges support from the Stanford Graduate Fellowship. D.L. acknowledges the Swiss National Science Foundation.

\section{Author contributions}

Y.L.C. and T.S. conceived the experiments. Y.L.C., Z.K.L. and J.A.S. carried out ARPES measurements with the assistance of S.K.M., D.H.L., R.G.M., M.K., D.L., S-L.Y. and P.S.K.; T.S. and M.K. synthesized and characterized bulk single crystals. H.J.Z. performed $a b$ initio calculations and X.L.Q. provided the theory support. All authors contributed to the scientific planning and discussions.

\section{Additional information}

Supplementary information is available in the online version of the paper. Reprints and permissions information is available online at www.nature.com/reprints. Correspondence and requests for materials should be addressed to Y.L.C. or T.S.

\section{Competing financial interests}

The authors declare no competing financial interests. 